\title{
Jugular venous ' $a$ ' wave in pulmonary hypertension: new insights from a Doppler echocardiographic study
}

\author{
Bojan B Stojnic, Stephen J D Brecker, Han B Xiao, Derek G Gibson
}

\begin{abstract}
Objective-To study the mechanisms underlying the dominant ' $a$ ' wave seen in patients with primary pulmonary hypertension.

Design-Retrospective and prospective examination of the jugular venous pulse recording, flow in the superior vena cava, and Doppler echocardiographic studies.
\end{abstract}

Setting-A tertiary referral centre for both cardiac and pulmonary disease, with facilities for invasive and noninvasive investigation, and assessment for heart and heart-lung transplantation.

Patients-12 patients with primary pulmonary hypertension, most being considered for heart-lung transplantation.

Results-Two distinct patterns of venous pulse and superior vena caval flow were identified: a dominant 'a' wave with no ' $v$ ' wave, an absent or poorly developed ' $y$ ' descent, and exclusively systolic downward flow in the superior vena cava (group 1, $\mathrm{n}=8$ ), and a dominant ' $v$ ' wave, deep ' $y$ ' descent and exclusively diastolic downward flow in the superior vena cava (group $2, n=4$ ). A comparison between the two groups showed age (mean (SD)) 42 (18) $v 36$ (7) years, RR interval $700(65) v 740$ (240) ms, left ventricular end diastolic dimension $3.6(0 \cdot 8)$ $v 3.2(1.0) \mathrm{cm}$ and end systolic dimension $2 \cdot 1(0.5) v 2.3(0.3) \mathrm{cm}$, right ventricular end diastolic dimension $2.6(0.5)$ v 2.8 $(0.6) \mathrm{cm}$, and pressure drop between right ventricle and right atrium 60 (8) $v$ 70 (34) $\mathrm{mm} \mathrm{Hg}$ to be similar. Duration of tricuspid regurgitation 520 (30) $v \quad 420$ (130) $\mathrm{ms}$ and the time interval of pulmonary closure to the end of the tricuspid regurgitant signal 140 (30) $v \quad 110$ (40) ms were longer in group 1 compared with group 2, whereas right ventricular filling time was much shorter 180 (70) $v$ 350 (130) ms. In seven patients from group 1, a single peak of forward tricuspid flow was present, but this pattern was seen in only one patient from group 2 .

Conclusions-In patients with primary pulmonary hypertension, the apparent ' $a$ ' wave seen in the venous pulse is, in fact, a summation wave. It is probably the result of large pressure changes that must underlie rapid acceleration and deceleration of blood across the tricuspid valve when the right ventricular filling time is short.

\section{(Br Heart J 1992;68:187-91)}

A dominant ' $a$ ' wave in the venous pulse is universally attributed in current textbooks of cardiology to an increase in the resistance to atrial emptying. ${ }^{1-3}$ In the absence of any abnormality at the level of the tricuspid valve, the primary abnormality is described as a reduction in right ventricular compliance. ${ }^{4}$ We found an entirely different mechanism underlying the dominant ' $a$ ' wave seen in a substantial minority of patients with dilated cardiomyopathy, in whom right ventricular filling time was greatly reduced by prolonged tricuspid regurgitation. ${ }^{5}$ A dominant 'a' wave, however, in such patients has not been widely recognised, at any rate since the work of Mackenzie, ${ }^{6}$ and there seemed to be no reason to suppose that such an unorthodox mechanism would be seen outside these rather special circumstances. Thus it seemed of interest to use similar echocardiographic, Doppler, and graphical methods to study an 'a' wave with well recognised associations, and to compare the underlying mechanisms with those seen in dilated cardiomyopathy. There was, we thought, no 'a' wave more typical in its genesis, than that associated with primary pulmonary hypertension.

\section{Patients and methods}

PATIENTS

We studied 12 patients with primary pulmonary hypertension, most of whom were being considered for heart-lung transplantation. Their ages ranged between 24 and 57 years and 10 were women. All were in sinus rhythm, and in all, the venous pressure was increased on clinical examination.

\section{MODE ECHOCARDIOGRAMS}

$M$ mode echocardiograms of the right and left ventricular cavities were made with reference to the cross sectional display on an ATL imager Mk 300I. The left parasternal view was used with the patients in the left lateral position. All $M$ modes were recorded photographically at a paper speed of $10 \mathrm{~cm} / \mathrm{s}$ on Honeywell equipment with simultaneous \\ Royal Brompton \\ Lung Hospital, Sydney \\ B B Stojnic \\ H B Xiao \\ Correspondence to: \\ Dr D G Gibson, Cardiac \\ Department, Royal
}


||||||||||||||||||||||||||||||||||||||||||||||||||||||||||||||||||||

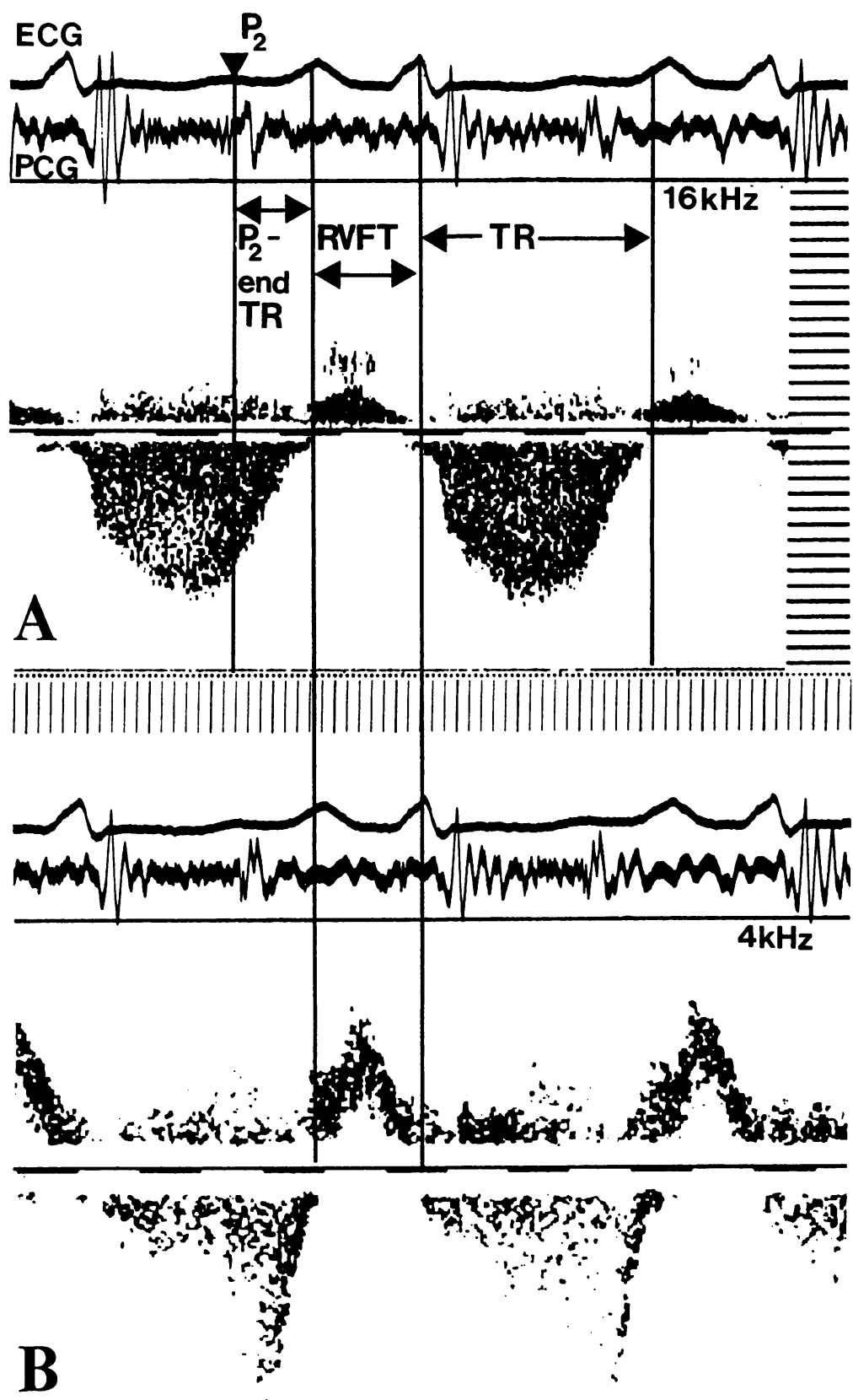

Figure 1 (A) Continuous wave Doppler recording of tricuspid regurgitation and $(B)$ pulsed wave Doppler recording of forward tricuspid flow. Both recordings are made with simultaneous electrocardiogram and phonocardiogram, and the Doppler calibrations are indicated. Pulmonary closure $\left(P_{2}\right)$ is indicated, and the time intervals of $P_{2}$ to the end of tricuspid regurgitation, right ventricular filling time, and tricuspid regurgitation duration are shown. ECG, electrocardiogram; PCG, phonocardiogram; $P_{2}$, pulmonary closure; $P_{2}$-end TR, time interval from pulmonary closure to the end of the tricuspid regurgitation signal; $R V F T$, right ventricular filling time; TR, tricuspid regurgitation duration.

\section{DOPPLER ECHOCARDIOGRAPHY}

Doppler recordings were made with Doptek equipment, and a $2 \mathrm{MHz}$ transducer. Retrograde flow across the tricuspid valve during systole was detected in continuous mode and forward flow velocities across the valve during diastole in pulsed mode (fig 1). Flow velocities in the superior vena cava were made from the right supraclavicular fossa in continuous mode with the transducer directed towards the right atrium. All recordings were made at a paper speed of $10 \mathrm{~cm} / \mathrm{s}$, with simultaneous phonocardiogram and electrocardiogram. From the record of tricuspid regurgitant flow, peak systolic frequency shift was obtained, and thus peak ventriculoatrial pressure drop was calculated from the modified Bernoulli equation. The time interval from $\mathrm{P}_{2}$ to the end of the regurgitant flow was taken as the relaxation time of the right ventricle, and the interval between the end of one pulse of tricuspid regurgitation and the start of the next as the filling time of the right ventricle (fig 1). On the pulsed Doppler record of forward flow, peak velocities during early diastole ( $E$ wave), and atrial systole (A wave) were noted. If only a single flow velocity pulse was present, with the start of early diastolic flow occurring after the $P$ wave of the succeeding beat, we referred to the filling pattern as summation. On the superior vena caval Doppler, we noted whether flow velocities towards the right atrium were greater during ventricular systole (' $x$ ' descent flow), or early diastole (' $y$ ' descent flow).

\section{JUGULAR VENOUS PULSE}

The jugular venous pulse was recorded with a Cambridge pulse transducer (time constant $4 \mathrm{~s})$ from the position on the neck where it was most apparent clinically. Where possible, simultaneous venous pulse records and records from the superior vena cava were made. The dominant wave, whether ' $a$ ' or ' $v$ ', was noted. In those patients with a dominant ' $v$ ' wave, the increase in the venous pulse above its minimum level at the start of the $Q$ wave of the electrocardiogram was noted, and expressed as a proportion of the total excursion during the cardiac cycle.

\section{STATISTICAL ANALYSIS}

Values are given as mean (SD). The possible statistical significance of differences between mean values was examined using Student's $t$ test, and $p<0.05$ was taken as significant.

\section{Results}

In eight patients (group 1), the jugular venous pulse showed a dominant ' $a$ ' wave (fig 2 ). In general, no ' $v$ ' wave at all was present, and the ' $y$ ' descent was either absent or poorly developed. In all these patients, downward flow in the superior vena cava occurred exclusively during ventricular systole, being complete before $P_{2}$. In the remaining four patients (group 2), there was a dominant ' $v$ ' wave in the venous pulse. Peak venous pressure occurred late during ventricular systole, and there was a the second heart sound, was routinely chect against the time of closure of the pulmonary valve, or the valve closure artefact at the end of pulmonary ejection on the pulsed Doppler record.

phonocardiogram and electrocardiogram. The internal dimensions of left and right ventricles the electrocardiogram. The identity of $P_{2}$, normally the start of the second component of 
Figure 2 Jugular venous pulse recording with simultaneous

electrocardiogram and phonocardiogram, with pulsed Doppler of superior vena caval flow. Note the dominant ' $a$ ' wave in the venous pulse with an ' $x$ '

descent and systolic

downward vena caval flow. $J V P$, jugular venous pulse; ECG,

electrocardiogram; PCG, phonocardiogram. Doppler calibration: full scale deflection $=2 \mathrm{kHz}$.
Figure 3 Jugular venous pulse recording with simultaneous

electrocardiogram and phonocardiogram, with pulsed Doppler of superior vena caval flow. Note the dominant ' $v$ ' wave in the venous pulse with a ' $y$ ' descent and diastolic downward vena caval flow. $J V P$, jugular venous pulse; ECG,

electrocardiogram; PCG, phonocardiogram. Doppler calibration: full scale deflection $=2 \mathrm{kHz}$.

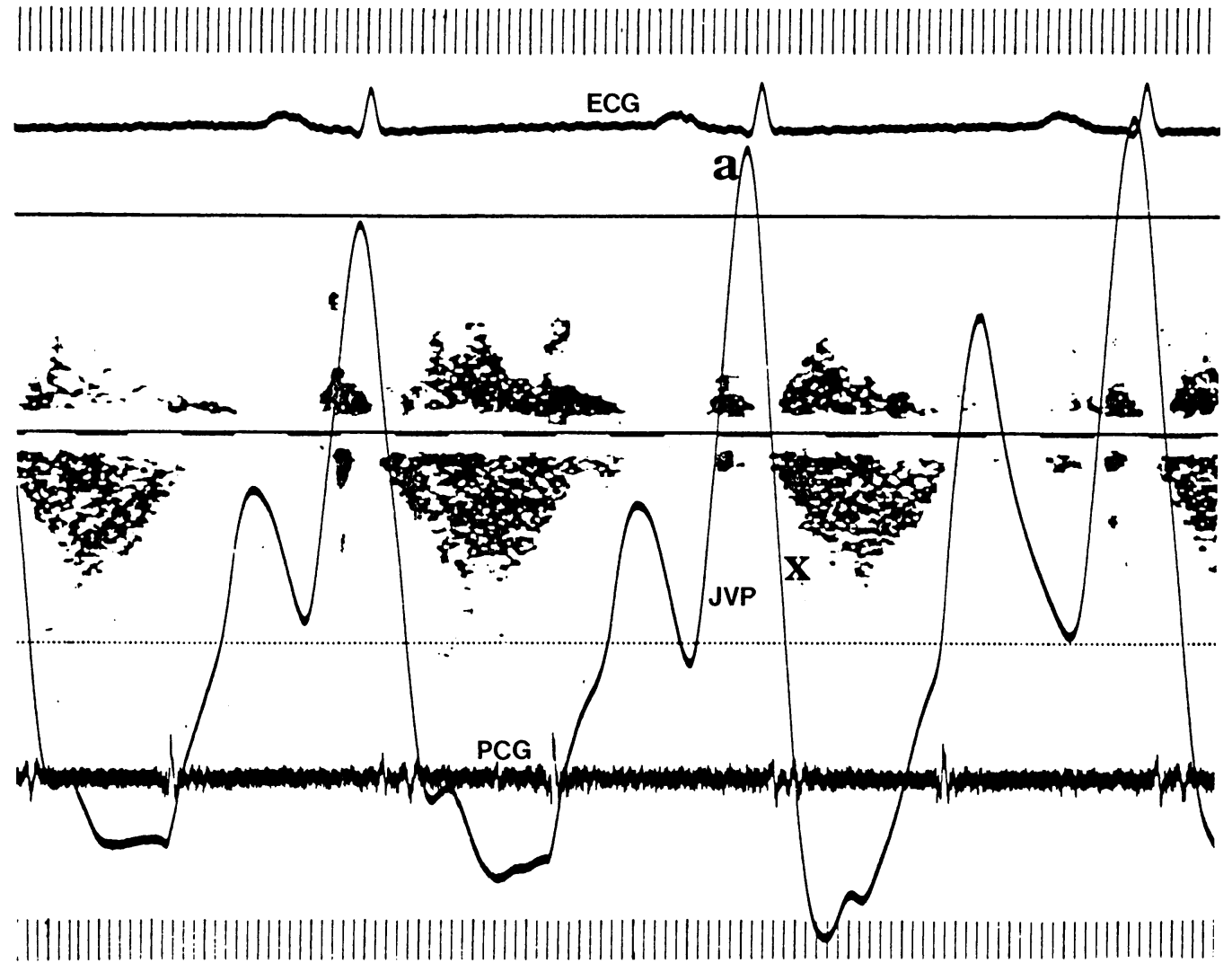

deep ' $y$ ' descent which started at about the same time as $\mathbf{P}_{2}$ (fig 3 ). In all these patients, flow from the superior vena cava towards the right atrium was found only during diastole. In three of these cases, the venous pressure rose mainly during systole, and in the fourth before the QRS complex of the electrocardiogram, suggesting a diastolic origin.
ASSOCIATION BETWEEN VENOUS PULSE AND RIGHT SIDED EVENTS

Because no patient had an intermediate pattern of either venous pressure or superior caval flow, those with a dominant 'a' wave (group 1) were compared with those with a dominant ' $v$ ' (group 2 and table). No significant difference was found between the two groups for age,

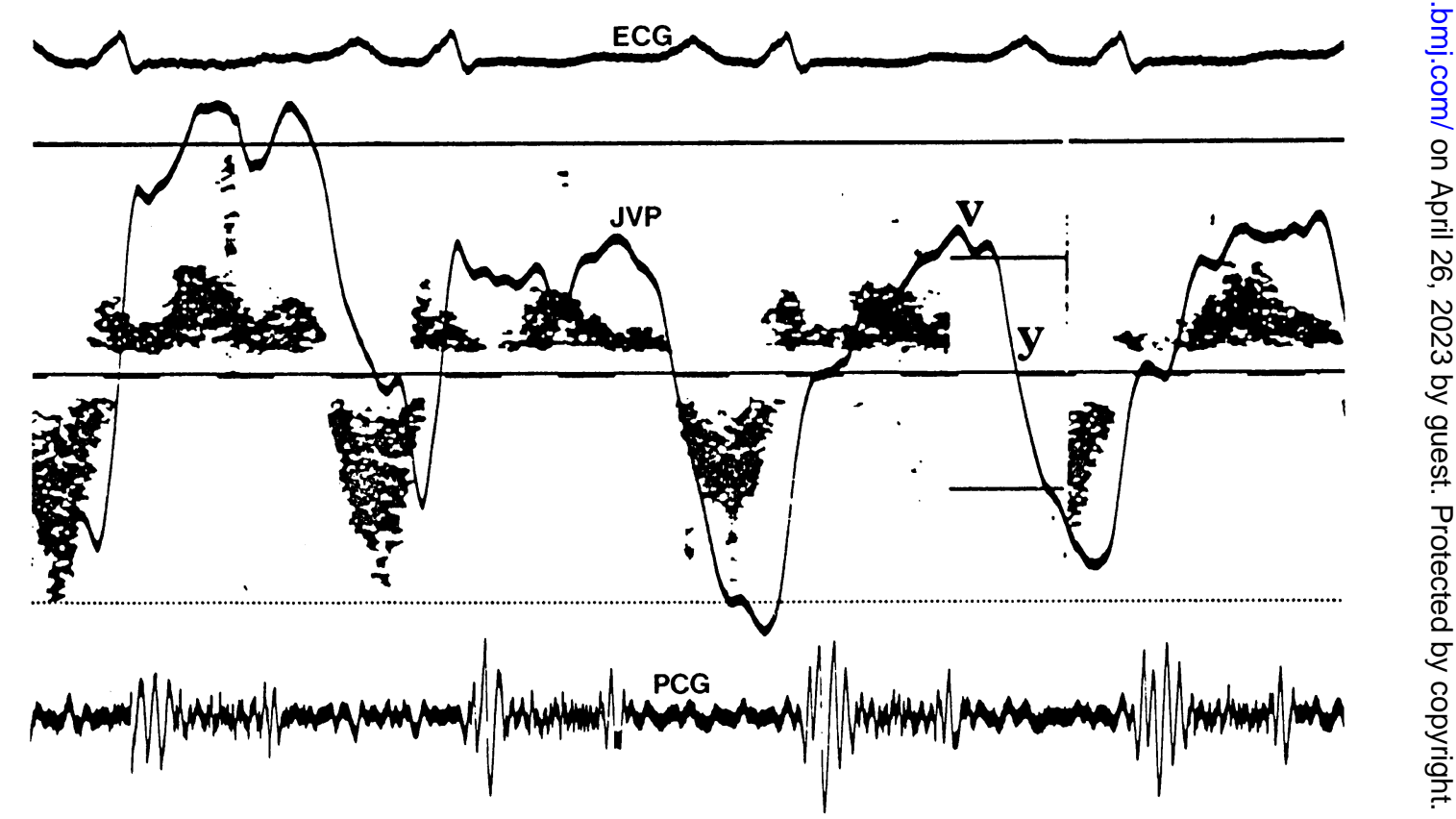


Jugular venous pulse pattern and associated characteristics (mean (SD)) in patients with primary pulmonary hypertension

\begin{tabular}{|c|c|c|c|c|}
\hline \multirow{2}{*}{$\begin{array}{l}\text { Variable } \\
\text { Age (y) } \\
\text { RR interval (ms) } \\
\text { LV EDD (cm) } \\
\text { LV ESD (cm) } \\
\text { RV EDD (cm) } \\
\text { RV-RA pressure drop (mm Hg) } \\
\text { Duration of tricuspid regurgitation (ms) } \\
\text { RV filling time (ms) } \\
\text { Pulmonary closure-end of tricuspid }\end{array}$} & \multicolumn{2}{|c|}{$\begin{array}{l}\text { Group } 1 \\
\text { Dominant ' } a \text { ' wave } \\
(n=8)\end{array}$} & \multicolumn{2}{|c|}{$\begin{array}{l}\text { Group } 2 \\
\text { Dominant ' } v \text { ' wave } \\
(n=4)\end{array}$} \\
\hline & $\begin{array}{c}42 \\
700 \\
3 \cdot 6 \\
2 \cdot 1 \\
2 \cdot 6 \\
60 \\
520 \\
180 \\
\end{array}$ & $\begin{array}{l}(18) \\
(65) \\
(0.8) \\
(0.5) \\
(0.5) \\
(8) \\
(30)^{\star} \\
(70)^{\star \star}\end{array}$ & $\begin{array}{c}36 \\
740 \\
3 \cdot 2 \\
2 \cdot 3 \\
2 \cdot 8 \\
70 \\
420 \\
350 \\
\end{array}$ & $\begin{array}{l}(7) \\
(240) \\
(1 \cdot 0) \\
(0 \cdot 3) \\
(0 \cdot 6) \\
(34) \\
(130) \\
(130) \\
\end{array}$ \\
\hline regurgitant signal (ms) & 140 & $(30)^{\star}$ & 110 & (40) \\
\hline
\end{tabular}

${ }^{\star} \mathrm{p}<0.05 v$ group $2 ;{ }^{\star \star} \mathrm{p}<0.01 v$ group 2 . EDD, end diastolic dimension; ESD, end systolic dimension; $L V$, left ventricle; $R A$, right atrium; $R V$, right ventricle.

heart rate (measured as RR interval), left or right ventricular transverse dimensions, or peak pressure drop from right ventricle to right atrium estimated from the peak velocity of tricuspid regurgitation, and no patient had diastolic tricuspid regurgitation. There were significant differences, however, in the relative timing of the systolic tricuspid regurgitation. In patients with a dominant ' $a$ ' wave, the whole regurgitant signal was more prolonged, as was that part after pulmonary valve closure (both p < 0.05), and even more significant, right ventricular filling time was little more than half that in patients with a dominant ' $v$ ' wave $(p<0.01)$. This difference was reflected in the forward tricuspid flow velocity pattern. In seven out of the eight patients with a dominant ' $a$ ' wave, there was only one peak, representing superimposed early diastolic and atrial components. By contrast, this pattern was seen in only one of those patients with a dominant ' $v$ ' wave, the rest having a dominant $\mathrm{E}$ wave.

\section{Discussion}

An increase in atrial activity is widely regarded as a homoeostatic mechanism, which maintains stroke volume in the presence of ventricular disease. Some disturbance of diastolic function-impaired compliance is often invokedcauses the component of stroke volume entering early in diastole to fall, and that during atrial systole, to be correspondingly increased. On the left side of the heart, this is associated with a dominant ' $a$ ' wave on the transmitral flow velocity trace, and often with a fourth heart sound. On the right side of the heart, exactly similar mechanisms have been invoked as the basis of the dominant ' $a$ ' wave often found in the venous pulse. ${ }^{124}$ The conditions thought to give rise to such an ' $a$ ' wave are found most typically in primary pulmonary hypertension. An overloaded right ventricle becomes hypertrophied; its diastolic properties are impaired, and in the absence of atrial or ventricular septa defect, increased right atrial activity shows itself as an increased pressure wave in the venous pulse. For these reasons, we took the condition as a paradigm of right ventricular overload to study interrelations between pressure and flow in more detail.

Our results confirmed these widely accepted ideas to the extent that a dominant presystolic wave is often present in the venous pulse of patients with primary pulmonary hypertension, but in almost every other respect they were incompatible. In seven out of the eight patients with such a wave, we found no evidence of reduced early diastolic flow across the tricuspid valve, nor a compensatory increase during atrial systole. Instead we found a single summation wave, in which early diastolic and atrial systolic components were superimposed. This pattern was the result of ventricular filling time being shortened to a mean value roughly half that seen in the rest of the patients, although heart rate was not significantly different. When filling time is short, stroke volume is only maintained if the velocity of blood flow into the ventricle is increased; this requires correspondingly high values of acceleration and deceleration. ${ }^{7}$ Acceleration of blood only occurs as the result of a pressure gradient. We suggest, therefore, that the presystolic pressure wave seen in the venous pulse of these patients is not the result simply of the effects of right atrial systole. Rather, it is the clinical manifestation of the pressure changes that must underlie the rapid acceleration and deceleration when filling time is short. It is thus better termed a summation wave, to stress its relation to the timing of intracardiac events.

When right ventricular filling time was longer, right sided events were different. The tricuspid forward flow velocity trace showed a dominant $E$ wave in three patients and a single wave in one; in no case was there a dominant ' $a$ ' wave. At the same time, the venous pulse showed a dominant ' $v$ ' wave and ' $y$ ' descent. These fundamental differences could not be attributed to age, heart rate, the size of the cavity of either right or left ventricle, or the drop in systolic pressure between right ventricle and right atrium. Rather, the reason why filling time was longer was that the overall duration of the tricuspid regurgitation was less. The main factor determining the duration of tricuspid regurgitation is peak right ventricular pressure $^{8}$; however, right ventricular disease might have further prolonged it in patients with a dominant ' $a$ ' wave, possibly by slowing relaxation. Alternatively, some process might have shortened the tricuspid regurgitation in patients with a dominant ' $v$ ' wave. On the left side of the heart, the duration of the mitral regurgitation falls as its severity increases, ${ }^{9}$ and we have previously suggested that the same process might act on the right side of the heart. ${ }^{10}$ In line with this explanation is the systolic form of the venous pulse in three of four patients, and the appearance of retrograde systolic flow in the superior vena cava (fig 3 ). We suggest, therefore, that in patients with a dominant ' $v$ ' wave, the tricuspid regurgitation was haemodynamically significant instead of being merely functional. The dominant $\mathrm{E}$ wave on the transtricuspid flow pulse does not necessarily reflect normal diastolic properties within the right ventricle; the striking drop in filling pressure associated with it strongly suggested that it had become normalised.

A characteristic chain of cause and effect can thus be seen to underlie a dominant presystolic 
wave in the venous pulse of patients with primary pulmonary hypertension. The pressure wave is the direct result of a summation filling pattern due to a short filling period requiring increased acceleration and deceleration rates of blood flow. Right ventricular filling time is short because functional tricuspid regurgitation is prolonged, and we have previously shown this prolongation to be the predictable consequence of a high systolic pressure in the right ventricle. ${ }^{8}$ When filling time is longer, and transtricuspid flow shows early diastolic and atrial components, the dominant ' $a$ ' wave is no longer present. Also, during the period when right ventricular tension is still decaying the mitral valve has already opened although the left ventricular filling pattern is disturbed, with reduced $E$ wave and increased A wave velocities. ${ }^{11}$ Thus the effect of atrial systole is indeed accentuated in patients with pulmonary hypertension, but on the left rather than on the right side of the heart. Finally, the mechanism underlying the presystolic jugular venous wave seen in our patients did, in fact prove to be similar to that previously described as occurring in patients with dilated cardiomyopathy. ${ }^{2}$ In such patients, however, right ventricular pressures were much lower, suggesting that the prolonged tricuspid regurgitation was caused by some other mechanism, possibly primary right ventricular disease.

This analysis suggests that the traditional ideas about right sided haemodynamics in pulmonary hypertension may have to be modified. At the same time, it stresses the scope of combined graphical and Doppler techniques in delineating disturbed physiology, and suggests new approaches to its pharmacological palliation. Whereas heart-lung transplantation may be the ultimate treatment for these patients, the severe shortage of donor material and the less satisfactory survival of patients after this operation than after isolated cardiac transplantation, both suggest a constructive role for delaying surgery by appropriate medical management.

SJDB is in receipt of a British Heart Foundation Junior Research Fellowship and HBX is supported by Brompton Hospital Special Cardiac Fund.

1 Craige E, Smith D. Heart sounds: phonocardiography; carotid, apex, and jugular venous pulse tracings; and ystolic time intervals. Jugular venous pulse. In Braunwald E, ed. Heart disease. A textbook of cardiovascular medicine. 3rd ed. Philadelphia: WB Saunders, 1988: $61-4$

2 Chatterjee K. Bedside evaluation of the heart: the physical examination. Abnormalities of the venous pulse and their significance. In: Parmley WW, Chatteriee K, eds Cardiology. Philadelphia: JB Lippincott, 1988:7-10.

3 O'Rourke RA. Physical examination of the arteries and veins (including blood pressure determination). Abnormal venous pulse. In: Hurst JW, Logue RB, Rackley CE ous pulse. In: Hurst JW, Logue RB, Rackley CE, et al, eds Hill, 1986:148-50.

4 Sutton GC. Examination of the cardiovascular system. Abnormalities of the venous pulse. In: Julian DG, Camm AJ, Fox KM, Hall RJC, Poole-Wilson PA, eds. Diseases of the heart. London: Baillière Tindall, 1989:108-10.

5 the heart. London: Baillière Tindall, 1989:108-10. dilated cardiomyopathy: sign of abbreviated right vendilated cardiomyopathy: sign of abbreviated righ
tricular filling time. Br Heart $J$ 1991;65:342-5.

6 Mackenzie J. The auricular form of the venous pulse. In: Mackenzie J, ed. The study of the pulse: arterial, venous, and hepatic and of the movements of the heart. Edinburgh: Young J Pentland, 1902:197-208.

$7 \mathrm{Ng} \mathrm{KSK}$, Gibson DG. Impairment of diastolic function by shortened filling period in severe left ventricular disease. Br Heart J 1989;62:246-52.

8 Brecker SJD, Xiao HB, Stojnic BB, Mbaissouroum M, Gibson DG. Assessment of the peak tricuspid regurgitan velocity from the dynamics of retrograde flow. Int Cardiol 1992;34:267-71.

9 Bradley JA, Gibson DG. Assessment of the severity of mitral regurgitation from the dynamics of retrograde flow. $\mathrm{Br}$

10 Strickland NH, Gibson DG. Reversed splitting of the second heart sound due to tricuspid regurgitation. Int $J$ second heart sound due

11 Stojnic BB, Brecker SJD, Xiao HB, Helmy SM, Mbaissouroum M, Gibson DG. Left ventricular filling characteristics in pulmonary hypertension: a new mode of ventricular interaction. Br Heart $J$ 1992;68:16-20. 